\title{
Rhus verniciflua Stokes against Advanced Cancer: A Perspective from the Korean Integrative Cancer Center
}

\author{
Woncheol Choi,, ${ }^{1}$ Hyunsik Jung, ${ }^{1}$ Kyungsuk Kim, ${ }^{1}$ Sookyung Lee, ${ }^{1}$ Seongwoo Yoon, ${ }^{1}$ \\ Jaehyun Park, ${ }^{1}$ Sehyun Kim, ${ }^{2}$ Seongha Cheon, ${ }^{3}$ Wankyo Eo, ${ }^{3}$ and Sanghun Lee ${ }^{1}$ \\ ${ }^{1}$ Integrative Cancer Center, East-West Neo Medical Center, Kyung Hee University, 149 Sangil-Dong, \\ Gangdong-Ku Seoul 134-727, Republic of Korea \\ ${ }^{2}$ Graduate School of East-West Medical Science, Kyung Hee University, Yongin 449-701, Republic of Korea \\ ${ }^{3}$ Department of Hematology and Oncology, East-West Neo Medical Center, Kyung Hee University, Seoul 134-727, Republic of Korea
}

Correspondence should be addressed to Sanghun Lee, integrative@korea.com

Received 29 July 2011; Revised 8 September 2011; Accepted 8 September 2011

Academic Editor: Masa-Aki Shibata

Copyright (๑) 2012 Woncheol Choi et al. This is an open access article distributed under the Creative Commons Attribution License, which permits unrestricted use, distribution, and reproduction in any medium, provided the original work is properly cited.

Active anticancer molecules have been searched from natural products; many drugs were developed from either natural products or their derivatives following the conventional pharmaceutical paradigm of drug discovery. However, the advances in the knowledge of cancer biology have led to personalized medicine using molecular-targeted agents which create new paradigm. Clinical benefit is dependent on individual biomarker and overall survival is prolonged through cytostatic rather than cytotoxic effects to cancer cell. Therefore, a different approach is needed from the single lead compound screening model based on cytotoxicity. In our experience, the Rhus verniciflua stoke (RVS) extract traditionally used for cancer treatment is beneficial to some advanced cancer patients though it is herbal extract not single compound, and low cytotoxic in vitro. The standardized RVS extract's action mechanisms as well as clinical outcomes are reviewed here. We hope that these preliminary results would stimulate different investigation in natural products from conventional chemicals.

\section{Introduction}

On September 6, 2008, the article of the title "We fought cancer... and cancer won" was in the Newsweek. The subtitle was that it is time to rethink the war on cancer, after billions spent on research and decades of hit or miss treatments. The improved understanding of molecular biology in cancer research has led to a number of new, effective treatments for cancer. However, the age-standardized death rate from all types of cancers combined first increased from 1970 to 1990 and then decreased through 2002, yielding a net decline of $2.7 \%$ [1]. Therefore, it is necessary to understand the cancer from a different point of view, which has been helped by the oriental medicine.

In view of the oriental medicine, mass or nodule is resulted from Qi and Blood stagnation. The development of a tumor could be interpreted as Qi Stagnation induced by stress, emotional disturbances, or external factors which could progress into Blood stagnation or stasis [2]. Traditionally Rhus verniciflua stoke (RVS) was considered to have the function of breaking up blood stasis and purging hardness [3]. Therefore, it could be applied for cancer treatment. RVS of the Anacardiaceae family, commonly known as the lacquer tree, was documented to be used for treating various stomach diseases, including tumor in East Asia including Korea at the 15 th century $[4,5]$. Recently several experimental studies showed that flavonoids from RVS have effective antiproliferative and apoptotic activities on various tumor cell lines including human lymphoma, breast cancer, osteosarcoma, and transformed hepatoma cells [4-7].

However, the clinical application of RVS has been limited because an allergenic component, urushiol, causes severe contact dermatitis in sensitive individuals $[8,9]$. Therefore urushiol, a mixture of several derivatives of catechol, should be removed from RVS for pharmaceutical use. A standardized extract of allergen-removed RVS was manufactured 
based on traditional method. Our integrative cancer center composed of oncologists and oriental medicine doctors was founded in June, 2006, and the standardized RVS extract was prescribed for the purpose of prolonging survival and improving the quality of life in patients with only palliative therapy available. Here, we review on the action mechanism and best outcomes of the standardized RVS extract with or without conventional treatment in patients with advanced or metastatic cancer.

\section{Preparations of Standardized Extract from RVS}

RVS stalk, which was 10 years old and grown in Wonju, Republic of Korea, was dried without exposure to direct sunlight and chopped up. The pieces were roasted in an iron pot at $240^{\circ} \mathrm{C}$ for 50 minutes to remove allergens and extracted two times with a 10 -fold volume of water at $90^{\circ} \mathrm{C}$ to $95^{\circ} \mathrm{C}$ for 6 hours (Korean patent no. 0504160). The extract was filtered with Whatman GF/B filter paper and concentrated under vacuum to remove water. The concentrate was lyophilized to a brownish powder. The extraction yield from $100 \mathrm{~g}$ of chopped material was $3.3 \mathrm{~g}$. A component analysis method using high-performance liquid chromatography showed that the RVS extract contained fustin, fisetin, sulfuretin, and butein, among others. The quality of the RVS extract was tested and controlled according to the quality standards of the Korea Food \& Drug Administration and our hospital's standards (fustin $>13.0 \%$, fisetin $>2.0 \%$, urushiol not detected). Daily oral administration is $1350 \mathrm{mg}$ (1 capsule containing $450 \mathrm{mg}$ three times a day) of RVS extract.

\section{Mechanism Studies}

3.1. Anti-Angiogenesis. The RVS was experimentally shown to have antitumor properties in carcinoma of breast and uterine cervix in vitro and suppress tumor volume in a xenograft mouse model system using A549 nonsmall cell lung cancer and Lewis lung cancer cells in vivo through inhibiting the proliferation and migratory activity of vascular endothelial growth factor $[10,11]$. It also significantly inhibited proliferation of human umbilical vein endothelial cells (HUVECs) induced by vascular epithelial growth factor (VEGF), despite its weak cytotoxicity against HUVECs.

Migration of endothelial cells toward a chemoattractant is an important step in angiogenesis [12]. When VEGF alone was present, the wound after scratching the cell monolayer was closed by cell migration. However, the addition of RVS significantly inhibited VEGF-induced migration of HUVECs similar to control cells (Figure 1). Maturation of migrated endothelial cells into a tube-like structure is a critical step for formation of functional vessels. HUVECs were seeded onto Matrigel and stimulated to form capillary networks with VEGF. As shown in Figure 2(b), robust and complete tube network formation was observed in VEGF-stimulated HUVECs. However, this effect of VEGF was significantly inhibited by RVS showing incomplete sprouting or branching or broken network between tubes of HUVECs (Figure 2).
These results indicate that RVS blocks migration and maturation of endothelial cells induced by VEGF.

3.2. Anti-Invasion. Matrix metalloproteinases (MMPs) are a family of zinc-dependent endopeptidases that remodel and degrade the extracellular matrix (ECM). Cancer cell migration and invasion of surrounding tissues are mediated in part by MMPs, especially MMP-2 and MMP-9 [13]. The effect of RVS on the invasiveness of human fibrosarcoma HT1080 cells was investigated by Boyden chamber assay [14]. The invasiveness of HT1080 cells was reduced in a dosedependent manner following 24-hour treatment of up to RVS $200 \mu \mathrm{g} / \mathrm{mL}$ (Figure 3). The ability of RVS extract to inhibit the secretion of MMP-2 and MMP-9 from HT1080 cells was studied. The levels of MMP-2 and MMP-9 were reduced by RVS, suggesting that the decrease in HT1080 cell invasion is a consequence, at least in part, of reduced activities of both MMP-2 and MMP-9. RVS inhibited MMP2 and MMP-9 activities with $\mathrm{IC}_{50}$ value of $1.01 \pm 0.07 \mu \mathrm{g} / \mathrm{mL}$ and $1.94 \pm 0.11 \mu \mathrm{g} / \mathrm{mL}$, respectively, by spectrofluorometric method which is very low concentration compared to other various herbs [15] (Figure 4).

\section{Clinical Studies}

4.1. Non-Small Cell Lung Cancer (NSCLC) [16]. The RVS was investigated to prolong survival in NSCLC after the failure of first-line or second-line chemotherapy. Forty patients treated with RVS for previously treated, advanced NSCLC between June 2006 and June 2009 were eligible for the final analysis. The median RVS administration period was 75 days. The median survival time was 8.4 months with a 1 -year survival of $40 \%$, and the disease control rate was $63.6 \%$, which is compatible with external controls in second-line chemotherapy. RVS showed more favorable outcomes in patients with better performance status or adenocarcinoma in terms of overall survival. The common RVS-related adverse events were mild epigastric pain and pruritis.

4.2. Colorectal Cancer (CRC) [17]. From July 2006 to November 2007, patients with conventional chemotherapy refractory metastatic CRC were checked. After fulfilling inclusion/exclusion criteria, 36 patients were eligible for the final analysis. Overall survival and adverse events of patients treated with RVS in the aftercare period were determined. The median RVS administration period was 2.7 months. The median overall survival for the entire population was 10.9 months (95\% confidence interval, 5.6-16.1) and 1-year survival rate was $44.4 \%$, which is compatible with external controls. By survival analysis using Cox proportional hazards model, the performance status and the prior chemotherapy regimen number significantly affected overall survival.

4.3. Pancreatic Cancer. Palliative chemotherapy-naïve patients with advanced or metastatic pancreatic adenocarcinoma were checked from July 2006 to June 2010. After applying inclusion/exclusion criteria, 42 patients were eligible for the final analysis. Overall survival, clinical benefit, and 


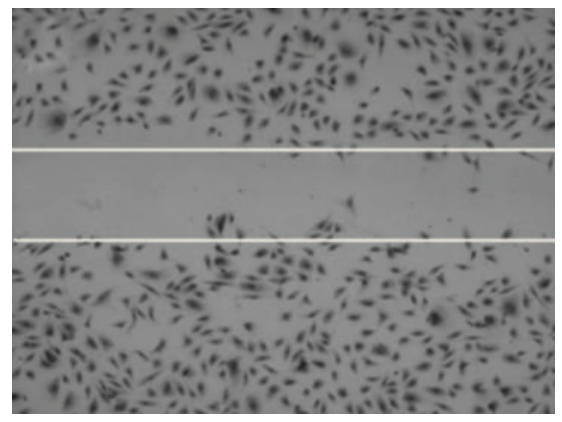

(a)

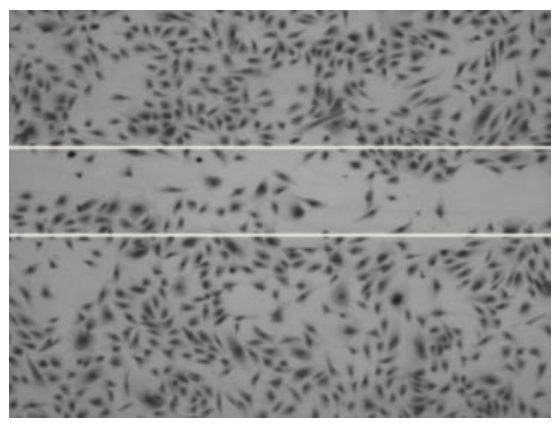

(c)

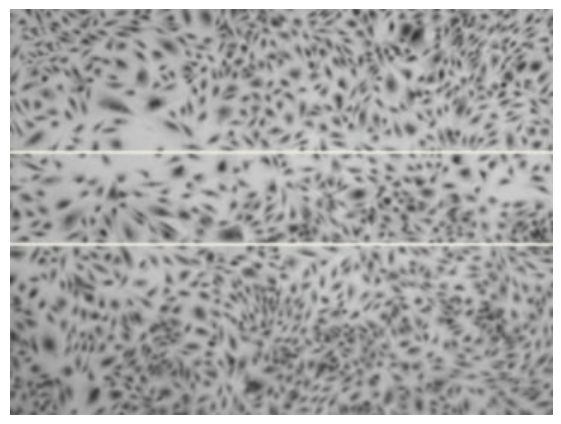

(b)

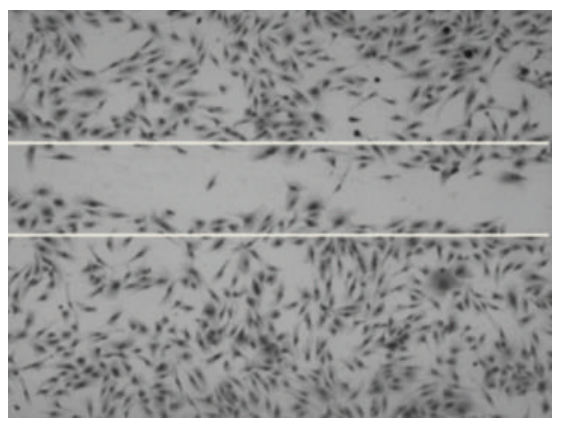

(d)

FIGURE 1: Effects of the standardized RVS extract on the migratory activity of VEGF-stimulated HUVEC (Wound-healing test). (a) The migrated cells in window-scraped field after scratching the cell monolayer; Control. (b) The wounds closed by cell migration from the wound edge after VEGF alone. (c) VEGF plus RVS $(50 \mu \mathrm{g} / \mathrm{mL})$. (d) VEGF plus RVS $(100 \mu \mathrm{g} / \mathrm{mL})$ showing inhibitory effect on migration compared to only VEGF-treated control.

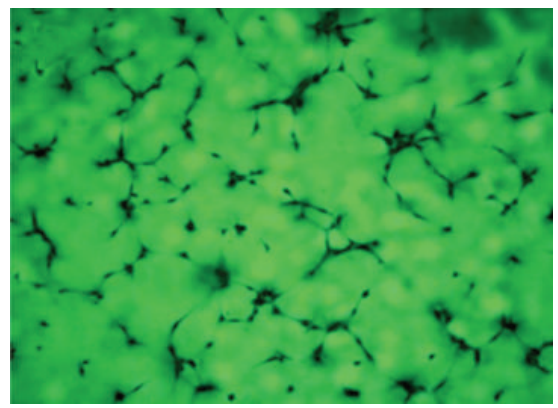

(a)

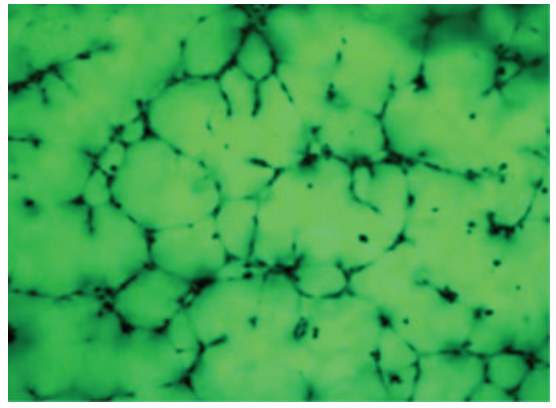

(c)

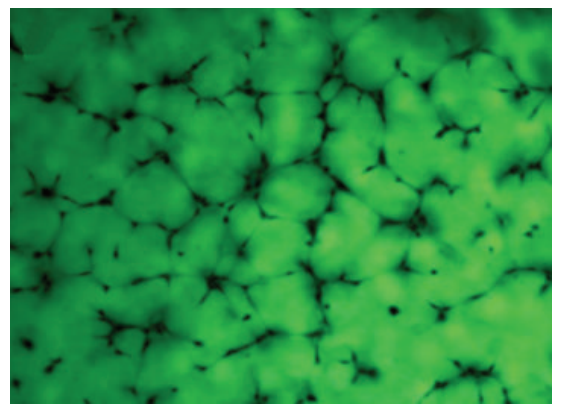

(b)

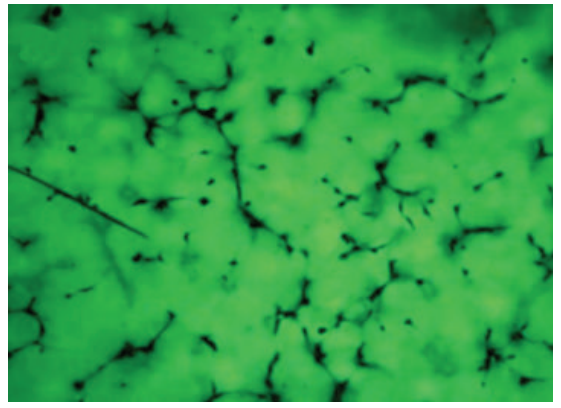

(d)

FIGURE 2: Effects of the standardized RVS extract on the tube formation in VEGF-stimulated HUVECs. (a) HUVECs seeding in Matrigel form capillary networks; Control. (b) The robust and complete tube network formation after VEGF alone. (c) VEGF plus RVS (50 $\mu \mathrm{g} / \mathrm{mL}$ ). (d) VEGF plus RVS $(100 \mu \mathrm{g} / \mathrm{mL})$ showing inhibitory effect on tube network formation compared to only VEGF-treated control. 


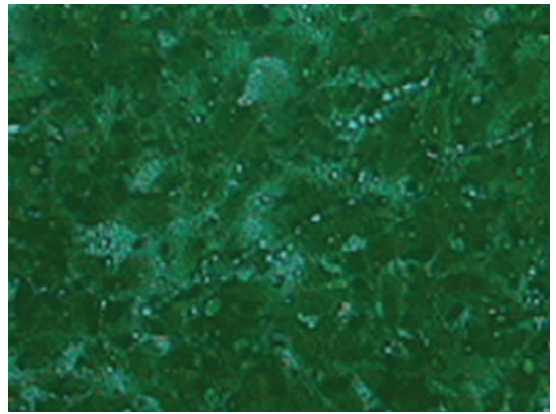

(a)

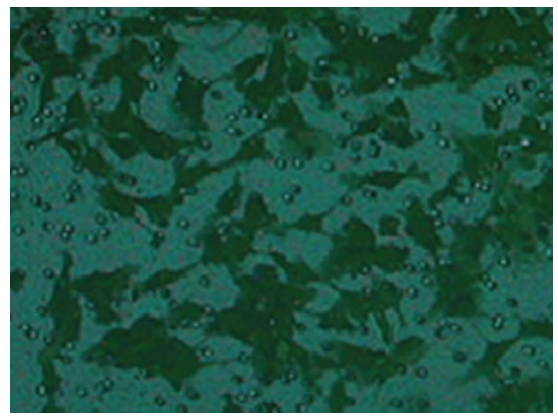

(c)

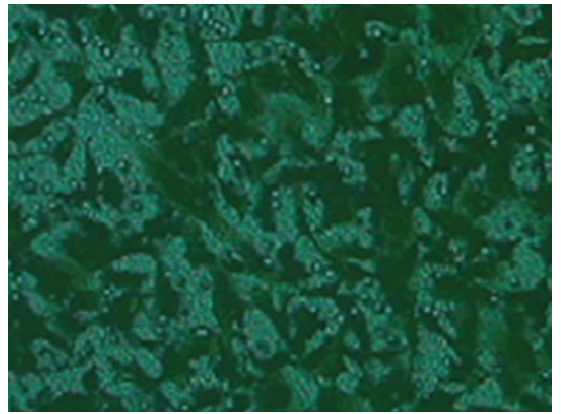

(b)

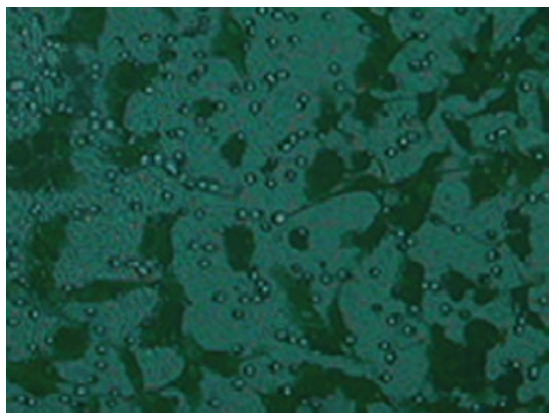

(d)

FIGURE 3: The effect of the standardized RVS extract on the invasiveness of human fibrosarcoma HT1080 cells using Boyden chamber assay. (a) Control. (b) RVS $50 \mu \mathrm{g} / \mathrm{mL}$. (c) RVS $100 \mu \mathrm{g} / \mathrm{mL}$. (d) RVS $200 \mu \mathrm{g} / \mathrm{mL}$ showing inhibitory effect on invasion compared to the control.

adverse events of these patients treated with RVS in the aftercare period were determined. The mean RVS administration period was 3.86 months (95\% confidence interval: $2.52-$ $5.20)$. The median overall survival for the entire population was 7.87 months, and 1-year survival rate was $26.2 \%$, which is compatible with external controls. By using univariate and multivariate analyses, RVS treatment including the performance status and prognostic index significantly affected overall survival. A clinical benefit response was also shown by RVS treatment which was not dependent on concurrent chemotherapy (Data not published).

\section{Case Studies}

5.1. Hepatic Cellular Carcinoma (HCC) [18]. A 62-year-old man underwent living donor liver transplantation for both hepatocellular carcinoma and end stage liver disease in March 2005. He had been diagnosed with hepatitis C infection in 1977. Unfortunately, the HCC recurred with multiple metastases in both lungs in September 2005, 6 months after liver transplantation. After systemic doxorubicin chemotherapy showed progression of lung metastases, he began receiving only RVS treatment in June 2006. CT scans 5 months later showed marked shrinkage of the lung metastases. Moreover, the patient tolerated the RVS well, without hematologic and nonhematologic toxicity. Four months later, metastatic mass in the right lower lobe of lung had increased in April 2007. The patient discontinued the RVS treatment and underwent radiation therapy. After finishing radiation therapy, he developed new liver metastases and died of progressive disease in November 2007.

5.2. Gastric Carcinoma [19]. An 82-year-old female was diagnosed with gastric cancer with a polypoid gastric mass approximately $25 \mathrm{~mm}$ in diameter at the middle body portion of the lesser curvature, a flat elevated lesion $50 \mathrm{~mm}$ in diameter at the prepyloric antrum, and the small gastrohepatic lymph nodes in October 2006. The endoscopic biopsy confirmed well-differentiated adenocarcinoma with a mutation in p53 that showed high nuclear activity of more than $80 \%$. Gastrectomy was not done because of old age and the concerns about the quality of life after surgery. RVS treatment was exclusively initiated on September 25, 2006. Five months later, the polypoid mass had markedly decreased and the flat elevated lesion had shrunk slightly, although the gastrohepatic lymph nodes were not changed. The biochemical parameters associated with liver and renal function were within the normal range, and no significant adverse effects from her RVS treatment have been observed. She maintains a good performance status up to now (July 2011).

5.3. Renal Cell Carcinoma (RCC) [20]. A 47-year-old man presented a $12 \mathrm{~cm}$ right renal mass and received a right radical nephrectomy which was pathologically the clear cell type of RCC in July 2006. Four months later, follow-up CT and PET scans revealed metastatic multiple pulmonary nodules. Palliative chemotherapy was recommended, but he refused it. 


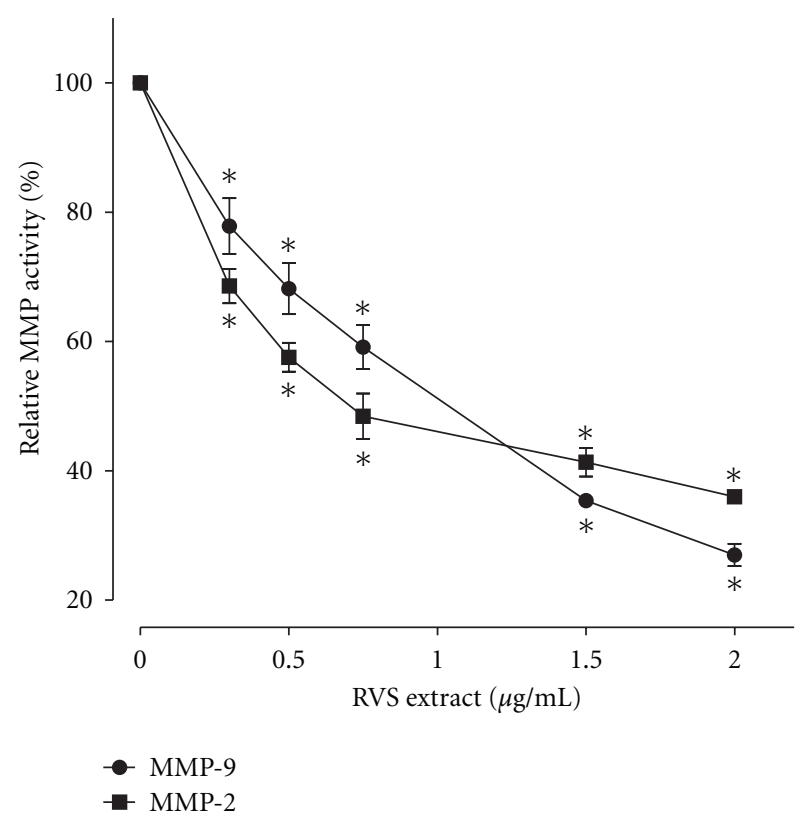

FIgURE 4: The effect of the standardized RVS extract on matrix metalloproteinase (MMP) activities by spectrofluorometric method. The $\mathrm{IC}_{50}$ of the standardized RVS extract for MMP-2 and MMP9 was $1.01 \pm 0.07 \mu \mathrm{g} / \mathrm{mL}$ and $1.94 \pm 0.11 \mu \mathrm{g} / \mathrm{mL}$ respectively. ${ }^{*} P<$ 0.05 versus respective controls.

Instead, only RVS treatment was initiated in December 2006. After 4 months, CT scans showed a complete response in all pulmonary metastases including resolution of right pulmonary artery thrombosis. Follow-up CT scans continued to demonstrate a complete response. He maintains a good performance status up to now (July 2011).

A 47-year-old man was diagnosed with the clear cell type of RCC accompanying multiple pulmonary nodules and underwent a radical nephrectomy for a $6.3 \mathrm{~cm}$ mass in September 2006. After cytoreductive surgery, the metastases in both lungs aggravated and a right adrenal mass was newly developed. After palliative sunitinib 2 cycles showed progression of lung metastases and both adrenal masses, he began receiving only RVS treatment in July 2007. After 9 months of RVS administration, chest CT scans showed the resolution of the masses, noted previously in the left upper lung. After 13 months of RVS therapy, CT scans showed significant reduction in the size of the metastatic masses in both adrenal glands. No evidence of disease continues in CT scans obtained up to now (July 2011).

5.4. NSCLC [21]. A 52-year-old female was diagnosed with pulmonary adenocarcinoma accompanying malignant pleural effusion confirmed by histologic examination in August 2006. Immunochemical staining pattern was a cytokeratin 7 (strong positive), cytokeratin 20 (negative), and thyroid transcription factor-1 (positive). One month later, CT scans showed an aggravation in malignant pleural effusion. She strongly refused recommended chemotherapy because of concerns about adverse effects. Instead, only RVS treatment was initiated in October 2006. After 1-month RVS treatment, CT scans showed marked decrease in pleural effusion and no interval change in mass. There was no significant change in tumor and pleural nodularity in a chest CT scans until January 2009. After progression of her disease, she was enrolled in a clinical trial (erlotinib) at other hospital in July 2009 and was lost to follow-up. Adverse effects from 34month RVS treatment were not observed.

\section{Discussion and Conclusion}

As several plants were used for cancer in traditional practice, rigorous in vitro and in vivo studies are essential and necessary to evaluate these extract's efficacy and safety before clinical trials, where the efficacy is dependent on the cancer-cell toxicity. Throughout this drug screening model such as the US National Cancer Institute (NCI) 60 human tumor cell line anti-cancer drug screen (NCI60) based on cytotoxicity, many drug developments could be possible from natural products [22]. In fact, half of all anti-cancer drugs approved internationally were either natural products or their derivatives and were developed on the basis of knowledge gained from small molecules or macromolecules that exist in nature [23]. However, it is questionable that the cell death is a critical end point for anti-cancer treatments. These days, the advances in the knowledge of cancer biology have developed some molecular-targeted agents for cancer treatment. Multiple tyrosine kinase inhibitors targeted on angiogenesis clinically prolong overall survival through cytostatic rather than cytotoxic effect to the cancer cells [24].

Korean health care system is different from other countries where two separate medical systems, western medicine and traditional Korean medicine, exist. The natural extract administered traditionally could be legally prescribed by traditional Korean medicine doctors. Therefore, it is possible for cancer patients to receive medication which might not be approved on conventional drug screen model because of low cytotoxicity. In fact, the RVS extract gave poor outcomes in NCI60 cell lines (Data not shown). However, some cancer patients in our center survived advanced cancer and are practically proven as responder to RVS extract. Except all previously described patients to be published, there are many responders in various cancer cell types including pancreatic cancer, cholangiocarcinoma, and sarcoma refractory to conventional chemotherapy. We suggest that standardized RVS extract could be a natural anticancer candidate from our experiences.

Single active constituents such as sulfuretin or butein have been characterized from RVS extract, following the conventional pharmaceutical paradigm of drug discovery $[25,26]$. Nevertheless, it is hypothesized that the active compound isolated from plants may rarely have high activity against cancer as the extract at comparable concentration without synergistic interaction or multifactorial effects between compounds present in herbal extracts [27]. Cancer is also complex disease having various biological capabilities such as sustaining proliferative signaling, evading growth 
suppressors, resisting cell death, enabling replicative immortality, inducing angiogenesis, activating invasion and metastasis, genome instability, tumor-promoting inflammation, deregulating energy metabolism, and evading immune destruction [28]. In response to targeted therapy, cancer cells may also reduce their dependence on a particular capability, becoming resistant to it. For example, even if potent angiogenesis inhibitors succeed in suppressing specific molecular targets, tumors adapt and shift from a dependence upon continuing angiogenesis to invasiveness and metastasis instead $[28,29]$. Therefore, multiple targeted agents with synergistic interaction are necessary to inhibit the cancer cell progression. The whole extract containing many constituents from natural products might make it possible to be more effective and less toxic in clinic than single potent compound. Based on clinical outcomes of RVS, it could be postulated to have various therapeutic targeting on cancer cell except the previously mentioned mechanism of action focused on angiogenesis.

In the era of targeted therapy, the predictive biomarkers have been identified to select patients more likely to benefit from it, which indeed makes cancer treatment personalized. In recent development of new drug, well-validated biomarker becomes more and more important from the excellent example of crizotinib targeting ALK mutation [30]. Unfortunately, there are no known clinically relevant biomarkers which could identify responders to the drugs targeted on angiogenesis [31]. Therefore, more research is needed for the predictive factors in order to find RVS extract-responders using molecular biology and traditional Korean medicine's diagnostic methodology. From our clinical results, effective outcome from RVS extract is not dependent on not only the cancer type but also the patient. Natural products are also known to affect immune function which could be monitored by immunomodulating cytokines such as IFN- $\gamma$, IL-2, and IL-10 [32]. Actually, there are reports on anti-inflammatory function of RVS or RVS flavonoids. Butein from RVS inhibits cytokine-induced nitric oxide production by suppression of inflammatory signaling in NF- $\kappa$ B pathways [33]. RVS hexane fraction and its major component, fisetin, significantly inhibit inflammatory cytokine production such as TNF- $\alpha$, IL-6, IL-8, and monocyte chemoattractant protein-1 [34]. RVS glycoprotein $(36 \mathrm{kDa})$ also has an inhibitory activity of T-helper type 2 (Th2) cytokines such as IL-4 and IL-10, which means to help immune dysfunction [35]. In aggressive pancreatic cancer, a cross talk between tumor cells and cancer-associated fibroblasts was reported to result in the induction of Th2-type inflammation which was well correlated with poor survival of patients [36]. Therefore, we try to focus cytokines-related immune system and find common genotype or phenotype backgrounds concerning drug metabolism in RVS extractresponders.

In conclusion, drug developments from natural products should be differently approached from the conventional pharmaceutical paradigm, and we hope that these preliminary results would stimulate further investigation into the effectiveness and tolerability of other natural products for the treatment of advanced cancers in clinical practice.

\section{Acknowledgment}

The authors would like to acknowledge the Kwanghyewon Medical Foundation for funding research that led to the understanding and writing of this paper.

\section{References}

[1] A. Jemal, E. Ward, Y. Hao, and M. Thun, "Trends in the leading causes of death in the United States, 1970-2002," Journal of the American Medical Association, vol. 294, no. 10, pp. 1255-1259, 2005.

[2] H. Beinfield and E. Korngold, "Chinese medicine and cancer care," Alternative Therapies in Health and Medicine, vol. 9, no. 5, pp. 38-52, 2003.

[3] A. Gamble, D. Bensky, and T. Kaptchuk, Chinese Herbal Medicine: Materia Medica, Eastland Press, Seattle, Wash, USA, 1993.

[4] Y. O. Son, K. Y. Lee, J. C. Lee et al., "Selective antiproliferative and apoptotic effects of flavonoids purified from Rhus verniciflua Stokes on normal versus transformed hepatic cell lines," Toxicology Letters, vol. 155, no. 1, pp. 115-125, 2005.

[5] H. S. Jang, S. H. Kook, Y. O. Son et al., "Flavonoids purified from Rhus verniciflua Stokes actively inhibit cell growth and induce apoptosis in human osteosarcoma cells," Biochimica et Biophysica Acta, vol. 1726, no. 3, pp. 309-316, 2005.

[6] M. Samoszuk, J. Tan, and G. Chorn, "The chalcone butein from Rhus verniciflua Stokes inhibits clonogenic growth of human breast cancer cells co-cultured with fibroblasts," BMC Complementary and Alternative Medicine, vol. 5, article 5, 2005.

[7] J. C. Lee, K. Y. Lee, J. Kim et al., "Extract from Rhus verniciflua Stokes is capable of inhibiting the growth of human lymphoma cells," Food and Chemical Toxicology, vol. 42, no. 9, pp. 1383-1388, 2004.

[8] T. H. Won, P. S. Seo, S. D. Park, D. L. Kim, and J. H. Park, "Clinical features in 147 patients with systemic contact dermatitis due to the ingestion of chicken boiled with Japanease lacquer tree," Korean Journal of Dermatology, vol. 46, no. 6, pp. 761-768, 2008.

[9] S. D. Park, S. W. Lee, J. H. Chun, and S. H. Cha, "Clinical features of 31 patients with systemic contact dermatitis due to the ingestion of Rhus (lacquer)," British Journal of Dermatology, vol. 142, no. 5, pp. 937-942, 2000.

[10] Z. Sminova, W. C. Choi, I. Kubasova, and A. Baryshnikov, "Antitumor efficacy of the allergen-removed extract (ACM909Q) in Rhus verniciflua," European Journal of Pharmaceutical Sciences, vol. 17, pp. 78-79, 2002.

[11] W. C. Choi, E. O. Lee, H. J. Lee et al., "Study on antiangiogenic and antitumor activities of processed Rhus verniciflua Stokes extract," Korean Journal of Oriental Physiology \& Pathology, vol. 20, pp. 825-829, 2006.

[12] M. H. Jung, S. H. Lee, E. M. Ahn, and Y. M. Lee, "Decursin and decursinol angelate inhibit VEGF-induced angiogenesis via suppression of the VEGFR-2-signaling pathway," Carcinogenesis, vol. 30, no. 4, pp. 655-661, 2009.

[13] M. Nguyen, J. Arkell, and C. J. Jackson, "Human endothelial gelatinases and angiogenesis," International Journal of Biochemistry and Cell Biology, vol. 33, no. 10, pp. 960-970, 2001.

[14] G. Moon, S. H. Lee, and J. H. Park, "Effect of allergen removed Rhus verniciflua extract on inhibition of tumor metastasis," Journal of Korean Traditional Oncology, vol. 15, no. 1, pp. 47$61,2010$. 
[15] J. S. Kim, B. Y. Park, E. K. Park et al., "Screening of antiangiogenic activity from plant extracts," Korean Journal of Pharmacognosy, vol. 37, no. 4, pp. 253-257, 2006.

[16] S. H. Cheon, K. S. Kim, S. Kim, H. S. Jung, W. C. Choi, and W. K. Eo, "Efficacy and safety of Rhus verniciflua stokes extracts in patients with previously treated advanced non-small cell lung cancer," Forschende Komplementarmedizin, vol. 18, no. 2, pp. 77-83, 2011.

[17] S. H. Lee, W. C. Choi, and S. W. Yoon, "Impact of standardized Rhus verniciflua stokes extract as complementary therapy on metastatic colorectal cancer: a Korean single-center experience," Integrative Cancer Therapies, vol. 8, no. 2, pp. 148-152, 2009.

[18] H. R. Kim, K. S. Kim, H. S. Jung, W. C. Choi, W. K. Eo, and S. H. Cheon, "A case of recurred hepatocellular carcinoma refractory to doxorubicin after liver transplantation showing response to herbal medicine product, Rhus verniciflua stokes extract," Integrative Cancer Therapies, vol. 9, no. 1, pp. 100$104,2010$.

[19] S. H. Lee, W. C. Choi, K. S. Kim, J. W. Park, and S. W. Yoon, "Shrinkage of gastric cancer in an elderly patient who received Rhus verniciflua Stokes extract," Journal of Alternative and Complementary Medicine, vol. 16, no. 4, pp. 497-500, 2010.

[20] S. K. Lee, H. S. Jung, W. K. Eo, S. Y. Lee, S. H. Kim, and B. S. Shim, "Rhus verniciflua Stokes extract as a potential option for treatment of metastatic renal cell carcinoma: report of two cases," Annals of Oncology, vol. 21, no. 6, pp. 1383-1385, 2010.

[21] S. H. Lee, K. S. Kim, W. C. Choi, and S. W. Yoon, "Successful outcome of advanced pulmonary adenocarcinoma with malignant pleural effusion by the standardized Rhus verniciflua stokes extract: a case study," Explore, vol. 5, no. 4, pp. 242-244, 2009.

[22] R. H. Shoemaker, "The NCI60 human tumour cell line anticancer drug screen," Nature Reviews Cancer, vol. 6, no. 10, pp. 813-823, 2006.

[23] D. J. Newman and G. M. Cragg, "Natural products as sources of new drugs over the last 25 years," Journal of Natural Products, vol. 70, no. 3, pp. 461-477, 2007.

[24] H. W. Hirte, "Novel developments in angiogenesis cancer therapy," Current Oncology, vol. 16, no. 3, pp. 50-54, 2009.

[25] D. G. Song, J. Y. Lee, E. H. Lee et al., "Inhibitory effects of polyphenols isolated from Rhus verniciflua on Aldo-keto reductase family 1 B10," BMB Reports, vol. 43, no. 4, pp. 268$272,2010$.

[26] J. H. Kim, C. H. Jung, B. H. Jang et al., "Selective cytotoxic effects on human cancer cell lines of phenolic-rich ethylacetate fraction from Rhus verniciflua Stokes," American Journal of Chinese Medicine, vol. 37, no. 3, pp. 609-620, 2009.

[27] H. Wagner and G. Ulrich-Merzenich, "Synergy research: approaching a new generation of phytopharmaceuticals," Phytomedicine, vol. 16, no. 2-3, pp. 97-110, 2009.

[28] D. Hanahan and R. A. Weinberg, "Hallmarks of cancer: the next generation," Cell, vol. 144, no. 5, pp. 646-674, 2011.

[29] F. Azam, S. Mehta, and A. L. Harris, "Mechanisms of resistance to antiangiogenesis therapy," European Journal of Cancer, vol. 46, no. 8, pp. 1323-1332, 2010.

[30] E. L. Kwak, Y. J. Bang, D. R. Camidge et al., "Anaplastic lymphoma kinase inhibition in non-small-cell lung cancer," New England Journal of Medicine, vol. 363, no. 18, pp. 1693-1703, 2010.

[31] A. M. Jubb, A. J. Oates, S. Holden, and H. Koeppen, "Predicting benefit from anti-angiogenic agents in malignancy," Nature Reviews Cancer, vol. 6, no. 8, pp. 626-635, 2006.
[32] G. Deng, H. Lin, A. Seidman et al., "A phase I/II trial of a polysaccharide extract from Grifola frondosa (Maitake mushroom) in breast cancer patients: immunological effects," Journal of Cancer Research and Clinical Oncology, vol. 135, no. 9, pp. 1215-1221, 2009.

[33] G. S. Jeong, D. S. Lee, M. Y. Song et al., "Butein from Rhus verniciflua protects pancreatic $\beta$ cells against cytokine-induced toxicity mediated by inhibition of nitric oxide formation," Biological and Pharmaceutical Bulletin, vol. 34, no. 1, pp. 97102, 2011.

[34] J. D. Lee, J. E. Huh, G. Jeon et al., "Flavonol-rich RVHxR from Rhus verniciflua Stokes and its major compound fisetin inhibits inflammation-related cytokines and angiogenic factor in rheumatoid arthritic fibroblast-like synovial cells and in vivo models," International Immunopharmacology, vol. 9, no. 3, pp. 268-276, 2009.

[35] J. Lee and K. T. Lim, "Plant-originated glycoprotein (36 kDa) suppresses interleukin-4 and-10 in bisphenol A-stimulated primary cultured mouse lymphocytes," Drug and Chemical Toxicology, vol. 33, no. 4, pp. 421-429, 2010.

[36] L. De Monte, M. Reni, E. Tassi et al., "Intratumor T helper type 2 cell infiltrate correlates with cancer-associated fibroblast thymic stromal lymphopoietin production and reduced survival in pancreatic cancer," Journal of Experimental Medicine, vol. 208, no. 3, pp. 469-478, 2011. 

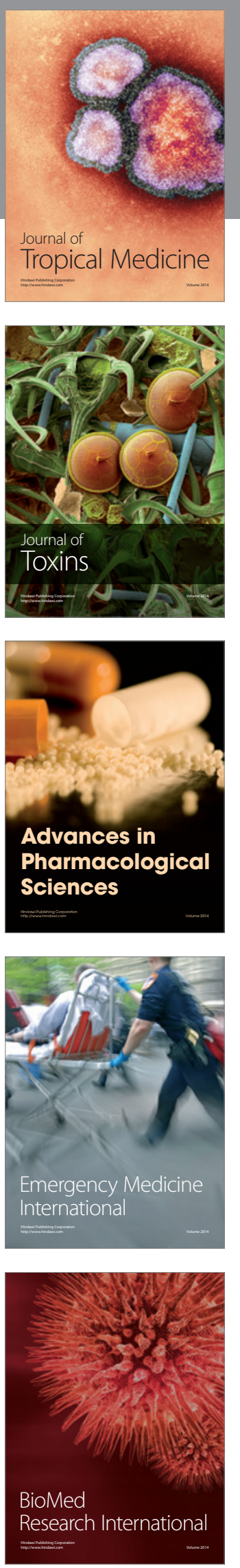
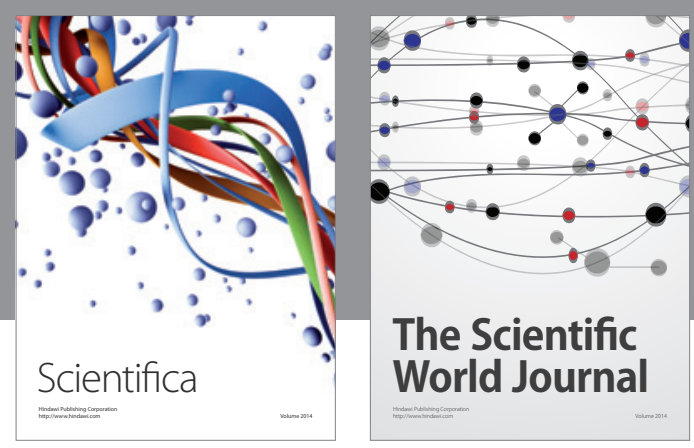

The Scientific World Journal
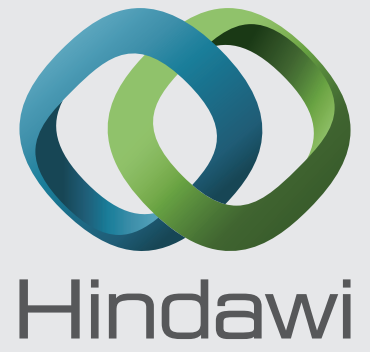

Submit your manuscripts at

http://www.hindawi.com
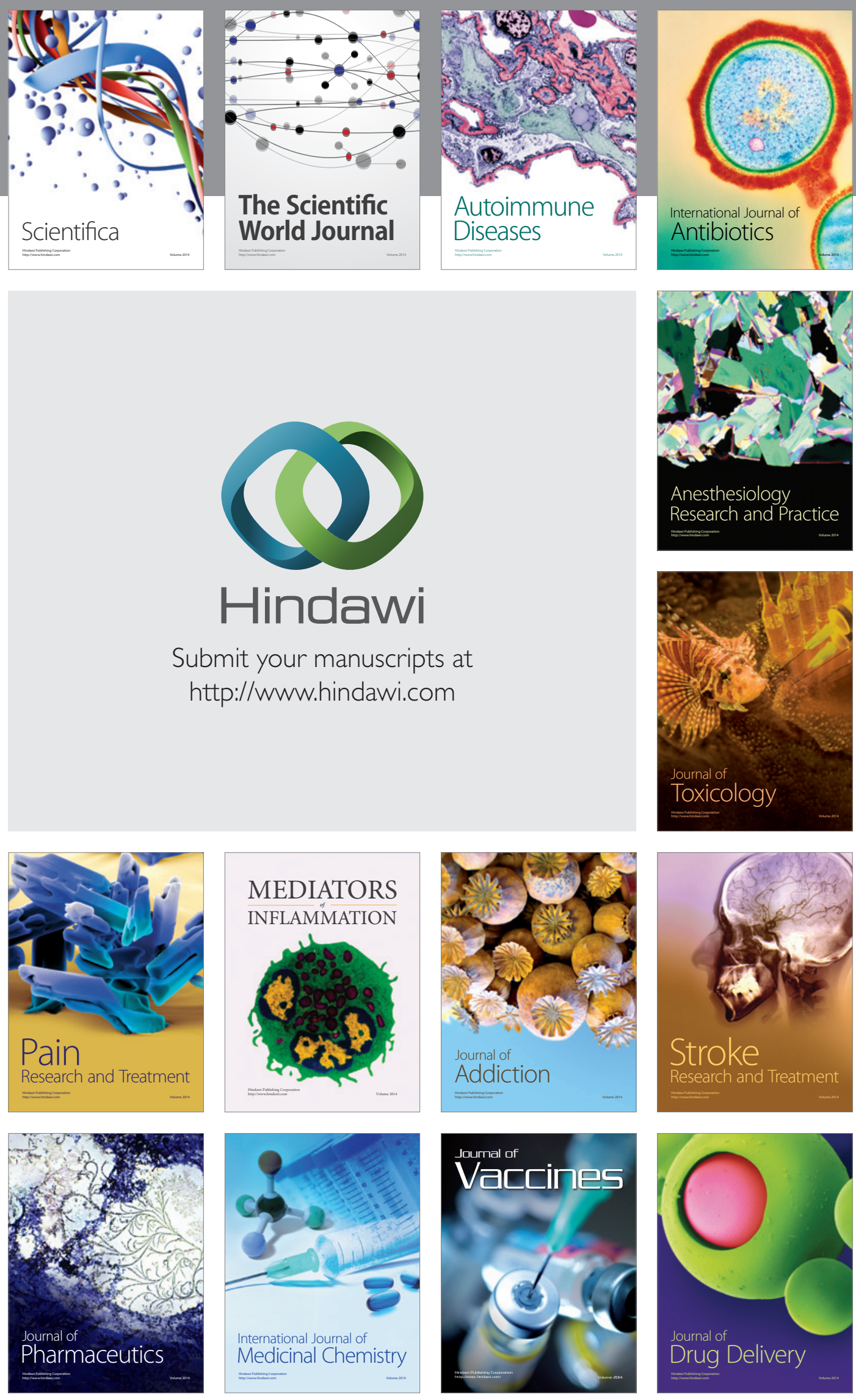